\title{
Adaptation to transients disrupts spatial coherence in binocular rivalry
}

\author{
Marnix Naber凶, Sjoerd Stuit, Yentl De Kloe, Stefan Van der Stigchel \& Chris L. E. Paffen
}

When one eye is presented with an image that is distinct from the image presented to the other eye, the eyes start to rival and suppress each other's image. Binocular rivalry leads to perceptual alternations between the images of each eye, during which only one of the images is perceived at a time. However, when the eyes exert weak and shallow suppression, participants tend to perceive both images intermixed more often. A recent study proposed that the precedence of mixed percepts positively correlates with the degree of adaptation to conflict between the eyes. However, this study neglected the role of visual transients, which covaried with the degree of conflict in the stimulus design. Here we report that not the conflict between the eyes but prolonged and repeated observations of strong visual transients cause participants to report more mixed percepts. We conclude that visual transients, such as sudden changes in contrast, draw attention, strengthen both eyes' image representations, and facilitate the adaptation to interocular suppression, which consequentially disrupts the spatial coherence in binocular rivalry. This finding is relevant to virtual- and augmented reality for which it is crucial to design stereoscopic environments in which binocular rivalry is limited.

Studying the dynamics of visual awareness with binocular rivalry. Binocular rivalry (BR) is a primary method in the scientific fields of cognitive psychology and neurosciences to study visual awareness. It consists of the presentation of separate images to each eye. When the two images are clearly distinct, the visual system is unable to fuse them into a coherent percept. Instead, the distinct mental representations from both eyes compete for priority to visual awareness. This results in the perception of unending perceptual alternations between the two images over time, a purely internally (mentally) driven process, since the physical environment is kept stable.

BR has been heavily exploited by psychologists, neuroscientists, and philosophers for a variety of reasons. One reason is that the dynamic properties of BR provide information on what type of images dominate more strongly or break into visual awareness faster (e.g., $\left.{ }^{1,2}\right)$. Such research is necessary to understand why people sometimes fail to notice objects (e.g., in traffic), how image-parts are grouped into ensemble objects (i.e., Gestalt principles), and why certain objects in the visual environment receive sensory priority (e.g., advertisements). BR is also the primary method used to study the interaction between the sensory processing of stimulus properties and other cognitive, high-level functions such as attention, numerosity, and emotions ${ }^{3-7}$. Furthermore, studies have revealed that a variety of brain regions and processes underlie changes in the content of visual awareness during $\mathrm{BR}^{8-11}$. Using $\mathrm{BR}$ to find the neural loci of consciousness and to identify the distinct processing stages of the stream of consciousness remains an ongoing line of research. Lastly, BR serves as a tool to examine to what degree information, that falls outside the scope of awareness, is processed and affects behavior ${ }^{12}$. Following the iceberg-mind analogy (i.e., most of what an iceberg's constitutes is submerged under water), most stimuli in a visual environment are not consciously perceived but may still have a deterministic effect on decision-making ${ }^{13}$. In sum, BR has been shown to be a valuable method to examine perceptual selection, the neurobiological underpinnings of awareness, and unconscious processing ${ }^{14}$. However, there is more to be learned from BR. While often overshadowed by discussions surrounding consciousness, BR also reflects how the eyes interact and strive for a stable, coherent percept. It is therefore necessary to understand under which circumstances dichoptic images fuse versus when they engage in binocular rivalry (e.g., ${ }^{15-18)}$. Especially now, with the rise of virtual and augmented reality goggles, it is of importance to understand how images can be best designed to prevent $\mathrm{BR}$, enhance the fusion of representations of both eyes, and create realistic depth perception. The experience of a coherent percept 
is important for effort-free viewing and the feeling of immersion when wearing stereoscopic goggles ${ }^{19}$. BR may therefore also be utilized to determine the level of "cooperation" between the eyes. It is important to note though that $\mathrm{BR}$, which a suppressive process, is phenomenally and mechanistically different from binocular fusion, which is a summative process ${ }^{20}$.

Exclusive versus nonexclusive, mixed episodes in binocular rivalry. How can BR serve as a tool to determine to what degree information of both eyes integrates rather than competes? To answer this question, it helps to focus on the spatio-temporal dynamics rather than merely the temporal dynamics of BR. Temporal dynamics include the rate at which switches in awareness occur and the ratio of left versus right eye dominance durations in perception. These measures indicate when and how often a change in awareness occurs and how strong, conspicuous, and relevant each image is to the visual system. Spatio-temporal dynamics reflect changes in local differences in eye dominance, and include episodes in which BR is in an intermediate, unstable state, in which perception exists of a mixture of the images of both eyes across image locations (i.e., piecemeal or non-exclusive rivalry). This latter measure indicates to what degree information of both eyes is integrated. However, this aspect of BR has received relatively little scientific attention, mainly because the dynamics of binocular rivalry are typically operationalized as stemming from a discrete on-off process (i.e., the image of the left or right eye is visible) by means of binary response options for the participants during testing (i.e., press either one of two buttons to report dominance of the two images). Only a handful of papers have looked at the non-binary properties of rivalry. For example, Naber et al. ${ }^{21}$ instructed participants to report mixed percept episodes of gratings, that moved in opposite direction in each eye, with a joystick. Participants reported the relative dominance of each grating through the degree of deflection of the joystick. It was observed that the timing and speed of the joystick deflections, reflecting the participant's perception of so-called traveling dominance waves, matched with the "objective" dynamics of the optokinetic nystagmus that was evoked by the moving gratings. Other studies also examined these traveling dominance waves, described best as the gradual emergence of a suppressed image that "flows" over the other, dominant image within a relatively short time $\operatorname{span}^{22-24}$. These waves tend to have a local starting point in the visual field and move with a certain velocity ${ }^{25-30}$, which are measures that also provide information about the relative priority of each image part. A few more studies inspected what type of images cause mixed percepts during rivalry ${ }^{31-33}$. For example, the more similar the images are across the eyes, the weaker the interocular suppression and the higher the chance of observing mixed rivalry ${ }^{32}$. Similarly, relatively similar gratings, with locally overlapping features, exhibit more mixed percepts as compared to complex and dissimilar objects such as houses and faces ${ }^{33}$. This means that when images mutually exert weak, shallow interocular suppression (i.e., a weak competition between the eyes) due to a local overlap of features between both eyes, exclusive (monocular) percepts are rarer and mixed episodes last longer. A recent adaptation study additionally showed that the durations of mixed episodes can be lengthened by first adapting participants to episodes of strong interocular conflict ${ }^{34}$. Interocular conflict was achieved by presenting gratings with tangential orientations to each eye (e.g., a horizontal grating in the left eye and a vertical grating in the right eye). The explanation for the increase in observations of mixed percepts after a prolonged period of interocular conflict is that the visual system includes neurons that detect interocular conflict between the eyes and strengthen interocular suppression depending on the degree of conflict ${ }^{35}$. Presumably, when these conflict detectors become less responsive to interocular conflict due to adaptation, interocular suppression becomes weak (i.e., shallow), resulting in more or longer episodes of mixed rivalry. However, adaptation to interocular conflict may not be the only plausible explanation for the reported effects on mixed percepts during rivalry. The current study investigates whether the weaker suppression (reflected by a larger incidence of mixed percepts) following adaptation in the study of Said and Heeger ${ }^{34}$ was due to adaptation to conflict, or whether other factors contribute to weaker suppression following adaptation.

Binocular conflict detectors versus visual transients. Although Said and Heeger ${ }^{34}$ elegantly applied the method of adaptation to support their model, which included conflict detectors, the authors may have overlooked the possibility that additional or alternative mechanisms drive the occurrence of mixed episodes. Here we propose that the presence of strong transients affects binocular rivalry and, in the context of the findings of Said and Heeger, could be the principal underlying factor for the facilitation of mixed percepts in rivalry after adaptation. To clarify, let us first describe how visual transients affect binocular rivalry: It is known that an intermittent stimulus presentation (i.e., interleaving content-rich image presentations with content-absent blanks) strongly reduces the alternation rate of binocular rivalry. Depending on the duration of the blank episodes, an image of one eye can remain dominant for minutes rather than seconds ${ }^{36,37}$. Such changes in the temporal domain of rivalry dynamics caused by strong on- and offset transients in the dichoptic display suggest that intermittent presentation enhances interocular suppression that is expressed as a strong suppression of the percept of the image from one of the eyes. Other types of visual transients, for example caused by local changes in luminance contrast or by eye-movements, can also strongly affect perception during (binocular) rivalry ${ }^{38-41}$. We here propose that strong visual transients may also affect spatio-temporal rivalry dynamics. As for the study of Said and Heeger $^{34}$, their conflict condition (producing strong adaptation) included an intermittent presentation paradigm while their weak adaptation condition did not (see Fig. 1, a modification of Figure 6 in Said and Heeger). In other words, the implementation of blanks, and thus of transient onsets and offsets of the images, may have directly affected interocular suppression rather than that the mere presence of interocular orientation conflict.

In three separate experiments we demonstrate that the presence of visual transients explains the degree of mixed percepts better than the presence of orientation conflict between the eyes. By manipulating the rate of transient changes in contrast and orientations during an adaptation period, we are able to show that these transients affect interocular suppression, resulting in decreased spatio-temporal stability of binocular rivalry and thus more mixed percepts. As so far previous rivalry studies only tested stimulus on- and offsets as transients, we will additionally compare the effects of different transient types. More specifically, we investigate how visual transients 
a

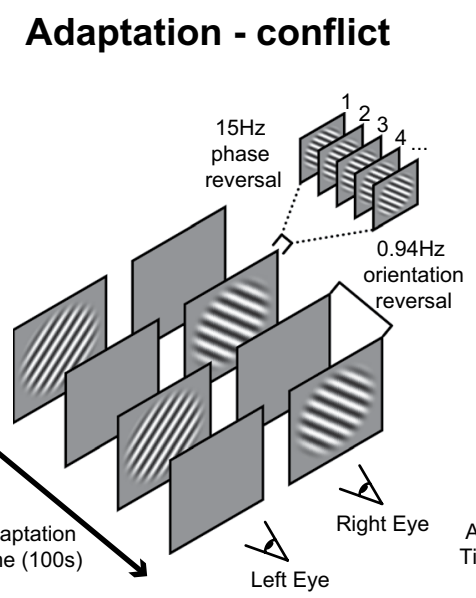

b

Adaptation - no conflict
C

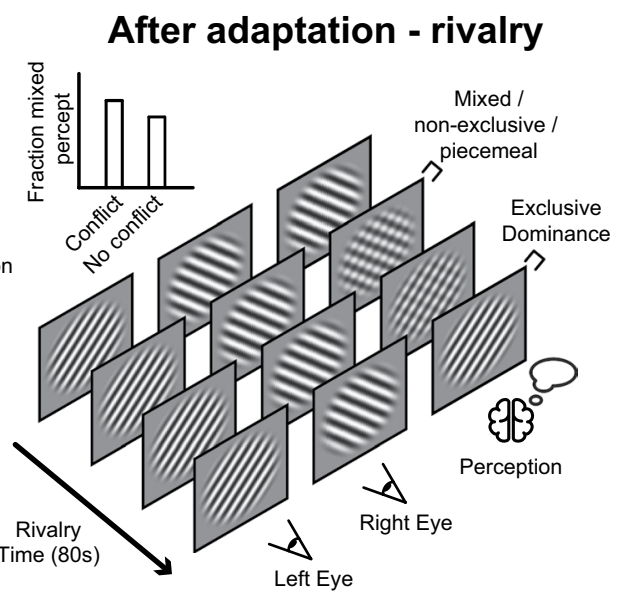

Figure 1. Procedural design by Said and Heeger. In the design of Said and Heeger's34, a single trial consisted of an adaptation period $(\mathbf{a}, \mathbf{b})$ that lasted for $100 \mathrm{~s}$ and a rivalry test period (c) that lasted $80 \mathrm{~s}$. Participants experienced regular binocular rivalry and indicated the onsets of exclusive and non-exclusive percepts with keyboard buttons during the subsequent test period (c). During the preceding adaptation period, participants passively viewed alternations in oriented gratings $(\mathbf{a}, \mathbf{b})$. The gratings' phase reversed at a rate of $15 \mathrm{~Hz}$ to prevent local brightness adaptation. More importantly, the perceived orientation alternated (counter-)clockwise at a rate of 0.94 times per second. According to Said and Heeger34, prolonged presentation of different orientations to the eyes (a) adapt opponency neurons that detect interocular conflict and drive interocular suppression (i.e., the degree the left eye's image is inhibited by the right eye's image and vice versa). However, when identical orientations are presented to both eyes at any point in time (b), they argue that no conflict between the eyes is present and neural conflict detectors do not adapt. Adaptation and therewith weaker interocular suppression subsequently leads to unstable perception, that is, a higher precedence of mixed (nonexclusive, piecemeal) percepts. A mixed percept consists of the presence of parts of two images from both eyes rather than a single exclusive image of one eye (c).

induced by (1) turning the (monoptic) display on and off - i.e. monocular contrast changes, (2) alternating the (monoptic) orientation of the displays - i.e. monocular (or binocular) orientation changes, and (3) perceived orientation changes - due to binocular rivalry, affect the spatio-temporal dynamics of binocular rivalry.

\section{Experiment 1}

Introduction. As described in the introduction, incoherent rivalry after adaptation (i.e., more mixed percepts) may be caused by the presence of transients, rather than the presence of interocular conflict. Such transients can be of any type, including changes in contrast and orientation. Here we extended the original conflict and no conflict conditions of Said and Heeger with novel conditions that either included or excluded the different transient types described above (see Fig. 2a; for details, see Stimulus and conditions), and investigated their individual contributions to the degree of mixed percepts following adaptation.

Methods. Participants. Twenty-six human individuals, all right-handed, young students (age: $M=23.4$, $S D=4.5 ; 21$ females) and with normal or corrected-to-normal vision, participated in Experiment 1. Participants were naïve to the purpose of the experiment, gave informed written consent before participation, and received either study credit or money (€6 per hour; Experiment lasted approximately 3 hours) after participation. The experiments conformed to the ethical principles of the Declaration of Helsinki and were approved by the local ethical committee of Utrecht University.

Apparatus. Stimuli were generated on two 24-inch ASUS VG248QE monitors (AsusTek, Taipei, Taiwan) with a dell computer (Dell, Round Rock, TX, USA) operating Windows 7 (Microsoft, Redmond, WA, USA) and MatLab version r2010a (Mathworks, Natick, MA, USA). The presentation monitors displayed 1920 by 1080 pixels at a $60-\mathrm{Hz}$ refresh rate. Each screen size was $53 \mathrm{~cm}$ in width and $30 \mathrm{~cm}$ in height ( 43 by 28 visual degrees), and the participant's viewing distance to the screen was fixed with a chin and forehead rest at $57 \mathrm{~cm}$. Each eye of a participant was presented with stimuli through a Wheatstone-inspired ${ }^{42}$ mirror stereoscope (for details, see ${ }^{43}$. Participants used the arrow buttons on a Logitech keyboard (Logitech International S.A., Lausanne, Swiss) to report their percept (left for exclusive dominance of counter-clockwise-oriented gratings, down for non-exclusive dominance, and right for exclusive dominance of clockwise-orientated gratings).

Stimuli and conditions. We used stimuli and conditions similar to those of Said and Heeger ${ }^{34}$ by including an adaptation phase (Fig. 1a,b) to affect perceptual stability in a subsequent rivalry phase (Fig. 1c). Stimuli had a $0.6^{\circ}$ radius in visual angle, a spatial frequency of $6.6 \mathrm{cycles} /{ }^{\circ}$, and edges softened by a cosine ramp of $0.1^{\circ}$ in width. To prevent ocular vergence responses and thus to promote binocular fusion (i.e., to achieve perception of two 


\section{Experiment 1}

a

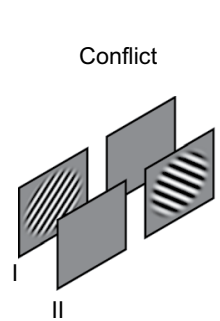

Adaptators

(shown before rivalry test period)
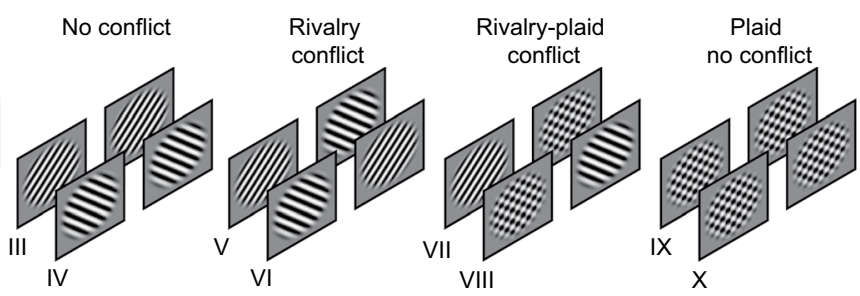

..... Monocular contrast $\quad-$ Perceptual orientation - Monocular orientation

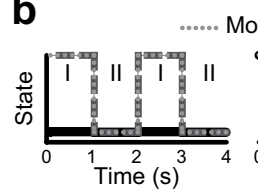

C

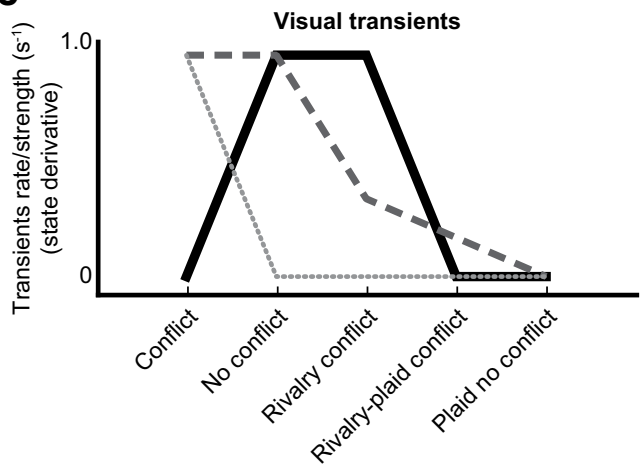

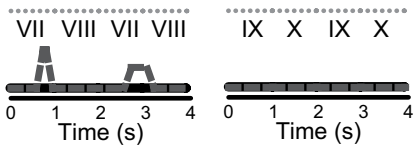

d

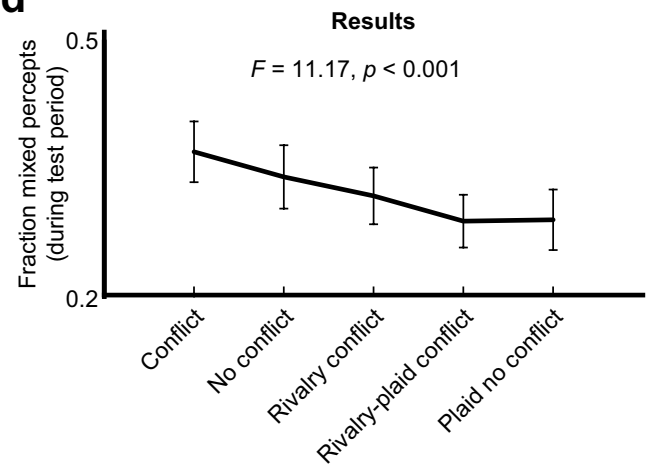

(shown after adaptation)

Rivalry

Figure 2. Adaptation conditions, transient profiles, predictions, and results of Experiment 1. Experiment 1 tested five adaptation conditions with different stimuli indicated with a separate roman number (a). Each condition induced changes in stimulus states, indicated with the corresponding roman numbers, as a function of time (b), including monocular (solid black) and perceptual orientation (dashed dark gray) transients, and contrast transients (dotted light gray). We included the original conditions of Said and Heeger in which orientation and contrast (first panel from left) or only orientation (second panel) changes at a rate of $0.94 \mathrm{~Hz}$ (solid black lines at (b)). We extended the original design by including a rivalry condition (third panel) with less frequently perceived orientation reversals (dashed dark gray lines at (b)) and a continuous orientation conflict between the eyes that excludes a monocular contrast conflict (dotted light gray lines at (b)). Another condition similar to the first panel was added but with a plaid rather than a blank screen in the other eye (fourth panel). A fifth condition with plaids presented to both eyes and thus no transients served as a baseline. Note that the perceptual orientation transients (dashed dark gray) are visible to the participant while the monocular transients (dotted light gray and solid black) are not (b). The pattern of expected fraction mixed percepts across conditions per feature (c) is based on the number and strength of transients within a normalized time interval (for legend, see panel b). The actual pattern of fraction mixed percepts as indicated by participants (d) did not perfectly match the patterns predicted by each individual adaptation type but matched a combination of monocular contrast and perceptual orientation factors (c).

spatially overlapping images), the stimuli were surrounded by a fusion stimulus. The fusion stimulus consisted of a $0.3^{\circ}$ wide annulus (not shown in the figures) with a random noise pattern that was identical for each eye's image and located at $2.25^{\circ}$ eccentricity.

Besides incorporating Said and Heeger's two original adaptation conditions in our design (first two panels from the left in Fig. 2a), we added an adaptation condition called "rivalry conflict" (panel three in Fig. 2a). In contrast to the conflict condition (panel one in Fig. 2a), this condition did not include monocular (within one eye) contrast transients (i) but did include monocular orientation transients (ii) and could potentially adapt opponency neurons due to the conflicting information between the eyes.

Note that both the conflict and no conflict condition produce clearly visible transients in orientation at a fixed rate of approximately one reversal per second. However, the rivalry conflict condition also induces alternations between the eyes at a rate dependent on the perception of the participant. This condition thus adds another research opportunity, namely to investigate to what degree the rate of perceived, binocular transients affect the stability of binocular rivalry. Therefore, to manipulate and weaken conflict between the eyes even further in an incremental manner, and therewith the rate of perceived orientation alternations, we added two more conditions with plaids (see panel four and five in Fig. 2a). These conditions serve as a baseline in which hardly any transients in terms of contrast and orientation are produced. 
While the rate of physical stimulus changes was kept constant at $0.94 \mathrm{~Hz}$ in the original two conflict and no conflict conditions, the three novel conditions were expected to differ in the number of evoked perceptual changes in orientation. Specifically, the rivalry conflict condition (third panel in Fig. 2a) should evoke perceptual rivalry as probed in the test phase. The rivalry-plaid conflict condition (fourth panel) should cause even fewer perceptual reversals because the images of the plaid and the oriented grating are typically merged in a single percept during binocular rivalry ${ }^{16}$. Note that the rivalry-plaid condition was similar to the original conflict condition of Said and Heeger but included the presentation of a plaid rather than a blank screen to the other eye as the oriented grating. The plaid no conflict condition (fifth panel) should cause no rivalry ${ }^{16,34}$. As the participants did not report alternation rates during the adaptation phase, authors $\mathrm{MN}$ and $\mathrm{YdK}$ independently confirmed that the orientation reversal rate and perceptual appearances were indeed manipulated as intended. In addition to the perceptual orientation transients, the five conditions also differed with regard to the presence of monocular contrast transients. Only the original conflict condition included intermittent blank presentations. The other four conditions thus contained no contrast transients (i.e., second-order, non-luminance based, contrast).

As shown in Fig. 2b, the frequency and strength of each type of stimulus change should differ considerably across the five conditions. For each type of stimulus change we plotted the derivative of the stimulus changes (Fig. 2c), indicating the strength of the visual transients and therewith the basis of a hypothetical pattern of results. For now we assume that each specific transient type independently affected the fraction mixed percepts during the test phase. Later in this paper we modelled weighted combinations of multiple transient types to investigate which of these best explain the fraction across all conditions and experiments (see last result section).

Procedure. The task for an participant was to attentively view the stimuli during the adaptation phase. Next they indicated their percept during the binocular rivalry phase as either exclusive (i.e., the majority of the surface of a single image was dominant) or mixed. The participants knew when to start reporting perceptions because the start of the rivalry test phase was marked by a sudden offset of phase reversals (i.e., the stimuli were contrast reversed at a rate of $15 \mathrm{~Hz}$ during the adaptation phase to prevent local brightness adaptation; see Fig. 1a,b). The participants kept their gaze on the fixation point at the center of the stimuli and screen.

Each condition was tested with six trials. The conditions were randomly interleaved across trials, and the trials were divided into two experimental sessions held at different days, because the experiment took more than 3 hours in total. Both sessions of the experiment started by having the participants align the stimuli on the screens to achieve best fusion, that is, the participants made sure the rivaling stimuli overlapped when viewed through the mirror stereoscope. Next, participants performed one rivalry test trial during which the contrast of the gratings was adjusted with the goal to counterbalance eye dominance by annulling between eye differences in dominance durations. Afterwards, the participants performed 30 trials and initiated the start of each trial with a button press.

Analysis. We refer to the independent variable as adaptation type. The dependent variable was the fraction mixed percepts, that is, the fraction of the total duration of perceptual episodes consisting of mixed dominance, as indicated by the participant. To investigate whether the factor adaptation type significantly affected the fraction mixed percept, we conducted a repeated measures (RM) ANOVA as a statistical test of significance (see figures for statistical outcomes) with Huynh-Feldt-Lecoutre (HFL) corrections if sphericity was violated. As post-hoc tests, we compared the fraction mixed percepts between each possible pair of conditions with two-tailed dependent t-tests (see tables in supplementary materials for statistical outcomes). We also examined the significance of effects of three transient types, namely that of (i) the presence versus absence of monocular contrast transients, (ii) the frequency of perceptual orientation transients, and (iii) the presence versus absence of monocular orientation transients. Post-hoc t-test results were not corrected for multiple comparisons because we only performed few, planned comparisons per experiment and all $p$-values were reported.

Results and discussion. We first aimed to test whether the adaptation type in the preceding adaptation phase affected the spatial stability of rivalry in the test phase. Indeed, the fraction mixed percepts during rivalry significantly varied across adaptation types (Fig. 2d; HFL-corrected RM-ANOVA: $F(3.08,76.88)=11.17$, $p<0.001)$. Qualitative inspection of the pattern of results suggested that the original conflict adaptation condition produced the highest fraction mixed percepts while the conditions with a plaid produced the lowest fraction.

Next, we determined whether we statistically replicated the findings by Said and Heeger ${ }^{34}$. While the direction of the effect appeared similar to these previous findings, the conflict and no conflict conditions did not differ significantly according to a two-sided t-test (see Supplementary table 1). A one-sided t-test, which can be argued to be appropriate in case of a prediction based on previous findings, did result in a significant effect $(t(25)=1.870$, $p=0.037)$. Not surprisingly, the fraction mixed percepts in the first half of the test phase, that is directly after the adaptation phase when effects of adaptation are typically strongest before fading off ${ }^{44}$, differed significantly between the conflict and no conflict condition, when tested with a two-sided t-test $(t(25)=3.726, p=0.001)$.

Next we continued to examine all conditions, including the novel three conditions, in order to determine which transient types drove the adaptation effects. When comparing the patterns of Figs. $2 \mathrm{c}, 2 \mathrm{~d}$, the decrease in perceptual orientations and monocular contrast across conditions matched the decrease in mixed percepts. To explore their individual significance of contribution to the pattern of results, we compared the effects of the presence versus absence of each transient type across conditions on the fraction mixed percepts. The first two conditions were the only conditions that included frequent and repetitive perceptual orientation transients and when pooled together (i.e., the fraction mixed percepts averaged across the two conditions) they produced significantly higher fraction mixed percepts than the other three conditions, which included less frequent to no orientation transients (Difference: $\mathrm{M}=0.054, \mathrm{SD}=0.051 ; t(25)=5.389, p<0.001$ ) The first conflict condition was the only condition that included monocular contrast transients and it produced significantly higher fractions mixed percepts than the other four conditions, which did not include monocular contrast transients (Difference: $\mathrm{M}=0.059$, 
$\mathrm{SD}=0.075 ; t(25)=3.986, p<0.001)$. The second and third conditions were the only conditions which included monocular orientation transients and they did not produce higher fractions mixed percepts than the other conditions without monocular orientation transients (Difference: $\mathrm{M}=0.013, \mathrm{SD}=0.044 ; t(25)=1.480, p=0.151$ ). Lastly, the first, second, and fourth conditions were the only conditions which included an orientation conflict between the eyes and they did produce higher fractions mixed percepts than the conditions without orientation conflict, but the effect was $\sim 50 \%$ weaker than that of perceptual orientation and monocular contrast transients (Difference: $\mathrm{M}=0.028, \mathrm{SD}=0.036 ; t(25)=3.908, p=0.001$ ).

To summarize the results of Experiment 1, the pattern of destabilization rates across all conditions is best explained by adaptation to both monocular contrast and perceptual orientation transients. Note that the third and fourth conflict rivalry(-plaid) conditions exhibited a conflict between the eyes but produced a lower fraction mixed percept than the first conflict condition. This latter finding cannot be explained by the conflict detector model of Said and Heeger ${ }^{34}$ because conflict was clearly present in the rivalry(-plaid) conditions, predicting an increase rather than the observed decrease in the fraction mixed percepts.

Because the manipulations of perceptual transients and monocular contrast (and conflict) were to some degree correlated across conditions, our next goal was to further disentangle the transient types and measure their individual contributions. As such, we continued to test the effects of monocular contrast transients independently from the other transient types in Experiment 2.

\section{Experiment 2}

Introduction. We have learned from Experiment 1 that it is likely that the presence of both perceptual orientation and monocular contrast transients in the adaptation phase disrupted the spatial coherency in binocular rivalry (i.e., increased the fraction mixed percepts) in the subsequent test phase. However, these two transients types co-varied across the conditions of Experiment 1. In Experiment 2 we manipulated the strength of contrast transients in isolation to further assess to what degree they contributed to incoherent perception during rivalry. We took a slightly different approach as compared to Experiment 1 by manipulating the contrast of the rivalling gratings rather than adding distracting information in the other eye. Based on the findings in Experiment 1, we predicted that a low, compared to a high, grating contrast leads to relatively weak monocular contrast transients during adaptation, eventually resulting in relatively weak adaptation and more coherent rivalry, as characterized by less mixed percepts.

Methods. All aspects of the methods were identical to Experiment 1, except for the participants, duration of the rivalry test phase, and adaptation type conditions. A new group of twenty individuals (age: $M=21.9, S D=2.3$; 14 females) participated in Experiment 2. The rivalry test phase was shortened from $80 \mathrm{~s}$ to $40 \mathrm{~s}$, because of the prominent effects of adaptation in the first $40 \mathrm{~s}$. We again included the original two adaptation conditions of Said and Heeger ${ }^{34}$ in the conditional design as a reference (see outmost left and right panel in Fig. 3a), as well as two novel conditions for which the contrast of tilted gratings were set at $50 \%$ and $25 \%$ (see second and third panel in Fig. 3a). These two conditions specifically affected the degree of perceptual orientation and monocular contrast transients (see dotted and dashed lines in Fig. 3b) and, based on the findings in Experiment 1, we predict that the decrease in contrast should weaken adaptation and decrease the fraction mixed percepts (Fig. 3c). Because of this prediction, we performed one-tailed instead of two-tailed post-hoc t-tests.

Results and discussion. The fraction mixed percepts differed across the four adaptation types at border significance (HFL-corrected RM-ANOVA: $F(2.42,46.05)=2.80, p=0.050$ ), showing a decreasing pattern across conditions (Fig. 3d; for post-hoc tests, see Supplementary Table 2). The original conflict adaptation condition produced the highest fraction mixed percepts $(M=0.43, S D=0.17)$ while the other conditions with a lower grating contrast or no monocular contrast produced significantly lower fractions $(\mathrm{M}=0.39, \mathrm{SD}=0.15 ; t(19)=$ $2.529, p=0.020)$.

Furthermore, the first conflict condition was the only condition that included $100 \%$ monocular contrast transients and it produced significantly higher fractions mixed percepts than the other three conditions (Difference: $\mathrm{M}=0.041, \mathrm{SD}=0.073 ; t(19)=2.529, p=0.020$ ). The first three conditions were the only conditions which included an orientation conflict between the eyes (and monocular orientation transients) and they did not produce higher fractions mixed percepts than the fourth condition without orientation conflict (Difference: $\mathrm{M}=0.022, \mathrm{SD}=0.066 ; t(19)=1.516, p=0.146)$.

In sum, we replicated the findings by Said and Heeger (i.e., a significant difference between the conflict and no-conflict conditions) and in addition observed that a weaker adaptation contrast decreased the occurrence of mixed percepts during rivalry. The pattern of results of Experiment 2 most closely matched the pattern predicted by the monocular contrast transients, although the flatter and higher pattern than in Experiment 1 suggested that adaptation was again driven by a weighted combination of perceptual orientation transients and monocular contrast transients (i.e., an average of the dotted and dashed line in Fig. 3c).

The results of Experiment 1 and 2 together favor a model that combines the effects of perceptual orientation and monocular contrast transients. It remains, however, unclear which of these transient types affects adaptation most. The final Experiment 3 was designed to extract the individual effects of monocular contrast versus perceptual orientation transients.

\section{Experiment 3}

Introduction. Experiment 3 disentangled the effects of monocular contrast transients and perceptual orientation transients by manipulating their presence and absence in opposite manners across conditions.

Methods. All aspects of the methods were identical to Experiment 2, except for the participants and adaptation type conditions. A new group of twenty human individuals (age: $M=21.4, S D=2.8 ; 16$ females) participated 


\section{Experiment 2}

a

Adaptation

(shown before rivalry test period)
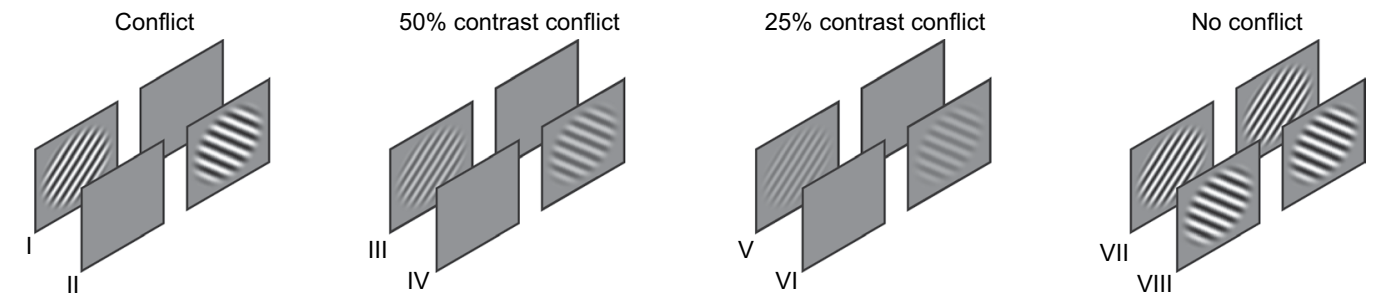

b

..... Monocular contrast
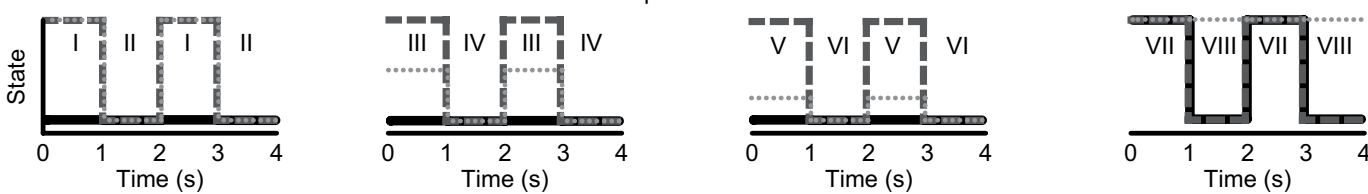

C

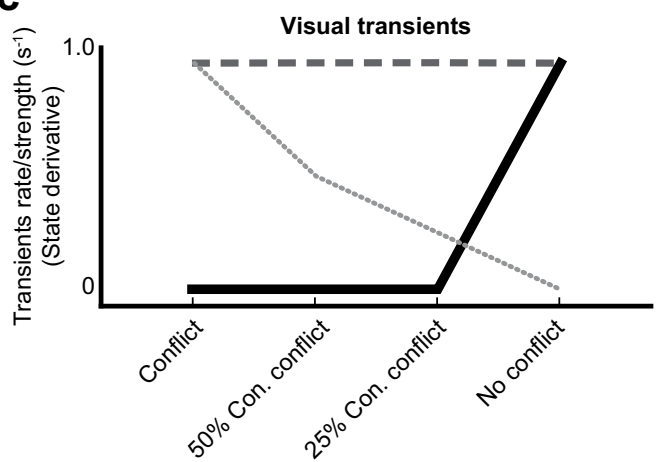

d

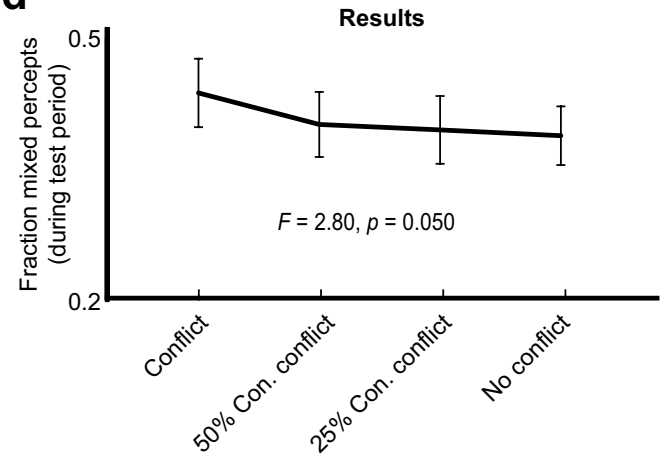

Figure 3. Adaptation conditions, transient profiles, predictions, and results of experiment 2. The design of Experiment 2 (a) contained two original adaptation (first and fourth panel from the left) conditions and two novel conditions (second and third panel), each with different time functions of monocular contrast (b). The new conditions mostly varied the strength of monocular contrast transients (c) and the pattern of results followed this manipulation (d).

in experiment 3. The original conflict condition of Said and Heeger again served as a baseline (first panel from the left in Fig. 4a) as well as the rivalry-plaid conflict condition from Experiment 1 (second panel in Fig. 4a). One novel condition consisted of a rivalry-plaid conflict condition in which the grating's contrast was lowered by $50 \%$ (see third panel in Fig. 4a). This manipulation created monocular contrast transients but decreased the frequency of perceived orientation reversals. If both transient types equally strong adapt interocular suppression, both factors should cancel each other and no difference is expected between the full and 50\% rivalry-plaid conflict.

We further disentangled the effects of perceptual orientation and monocular contrast transients by solely removing perceptual orientation transients in the last condition (see fourth panel in Fig. 4a). This condition consisted of the presentation of a single, non-rotating tilted grating that switched between eyes over time.

The latter three conditions affected the degree of perceptual orientation and monocular contrast transients in opposite manners (see lines in Fig. 4b) and each transient type predicted a different pattern of results (Fig. 4c).

Results and Discussion. The fraction mixed percepts significantly differed across the four adaptation types (HFL-corrected RM ANOVA: $F(2.19,41.55)=9.20, p<0.001$ ), showing a U-shaped pattern across conditions (Fig. 4d). The original conflict adaptation condition produced the highest fraction mixed percepts, the rivalry-plaid conflict and single orientation no conflict conditions scored medium fractions, and the $50 \%$ rivalry-plaid had the lowest fraction (for post-hoc tests, see Supplementary Table 3). The pattern of results most closely matched a pattern predicted by the combination of perceptual orientation and monocular contrast transients. However, the effects of weaker perceptual orientation transients and stronger monocular contrast transients in the $50 \%$ as compared to $100 \%$ rivalry-plaid condition did not cancel each other out. In fact, the $50 \%$ contrast rivalry-plaid condition resulted in a significantly lower fraction mixed percepts than the $100 \%$ contrast rivalry-plaid condition $(t(19)=1.787, p=0.045)$, indicating that the weakening of perceptual orientation transients had a stronger effect than the strengthening of the monocular contrast transients. In line with this finding, the full removal of perceptual orientation transients with the single orientation no conflict condition decreased the fraction mixed percepts (compared to conflict condition: $M=0.10, S D=0.14$ ) to a similar degree as the removal of half the monocular contrast transients $(\mathrm{M}=0.07, \mathrm{SD}=0.10 ; t(19)=1.524, p=0.144)$. 


\section{Experiment 3}
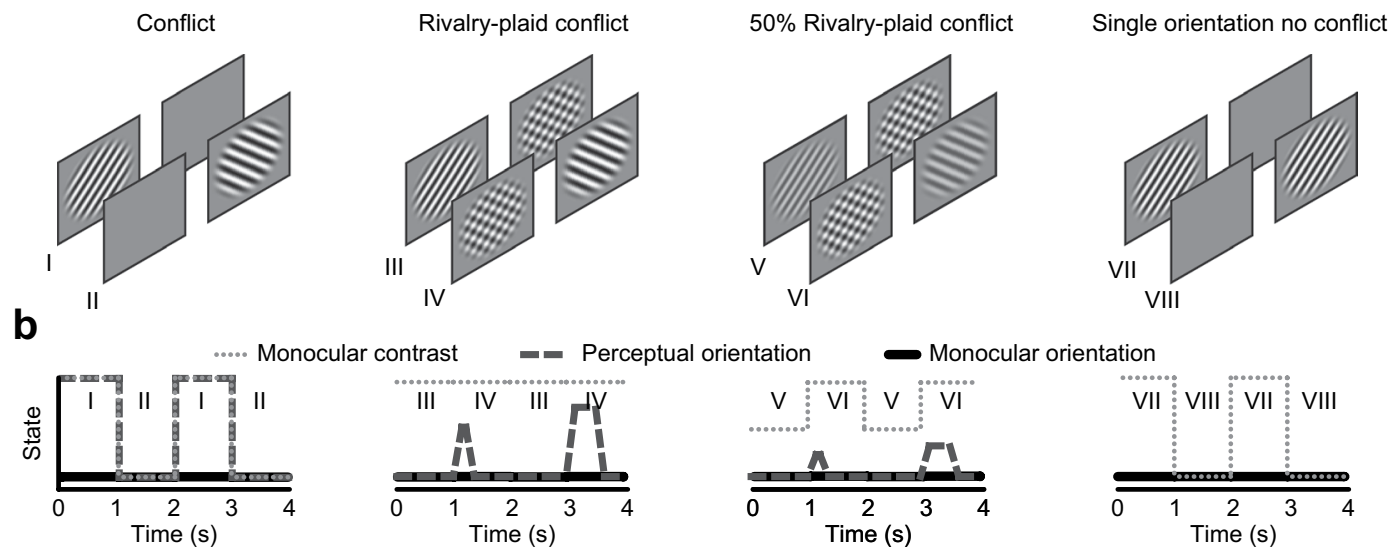

.... Monocular contrast $\quad$ - Perceptual orientation
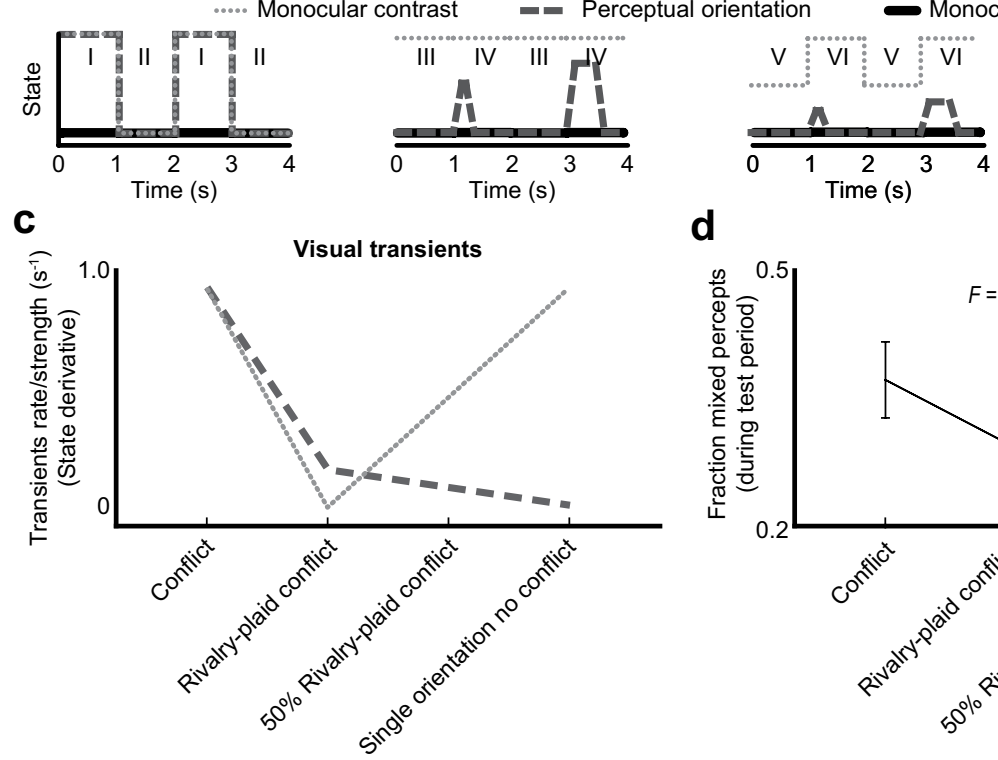

d
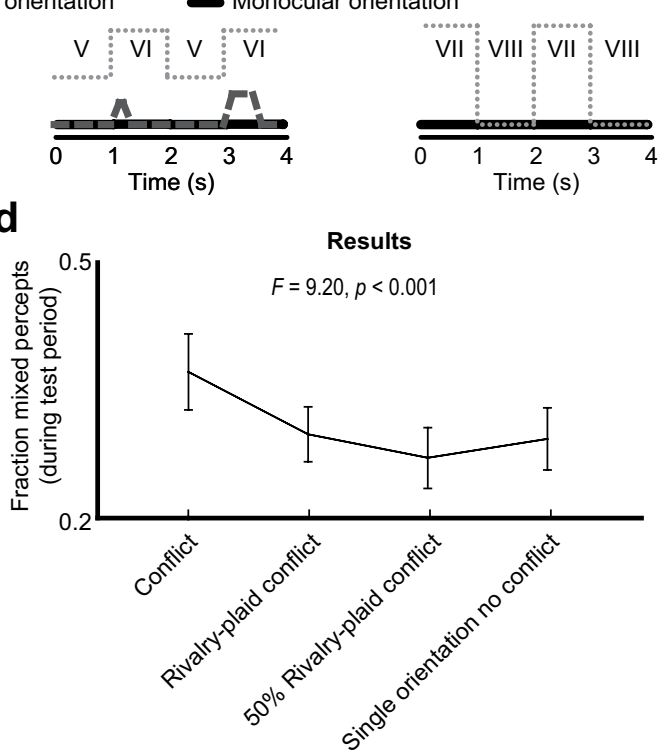

Figure 4. Adaptation conditions, transient profiles, predictions, and results of Experiment 3. Same plots as in Figs. 2 and 3 but now for Experiment 3 with two novel conditions (panel 3-4 at plots (a) and (c)). The pattern of results again reflected a combined weight of monocular contrast and perceptual orientation transients $(\mathbf{c}, \mathbf{d})$.

Furthermore, the first and fourth condition were the only conditions that included $100 \%$ monocular contrast transients and they produced significantly higher fractions mixed percepts than the other two conditions (Difference: $\mathrm{M}=0.045, \mathrm{SD}=0.066 ; t(19)=3.045, p=0.007$ ). The first condition was the only conditions that included frequent perceptual orientation transients and it produced significantly higher fractions mixed percepts than the other conditions (Difference: $\mathrm{M}=0.080, \mathrm{SD}=0.100 ; t(19)=3.584, p=0.002$ ). The first three conditions were the only conditions which included an orientation conflict between the eyes and they did not produce higher fractions mixed percepts than the condition without orientation conflict (Difference: $M=0.020, S D=0.048 ; t(19)$ $=1.817, p=0.085$ ).

In sum, the results of Experiment 3 suggest that mainly adaptation to perceptual orientation transients and to some extent adaptation to monocular contrast transients cause higher fractions of mixed percepts, indicating more non-exclusive dominance and spatially incoherent rivalry. Note again that almost all conditions included orientation conflict but did not produce similar fractions of mixed percepts. This is in contrast with suggestions by Said and Heeger ${ }^{34}$.

\section{Model - Weighted combinations of transient types}

The patterns of results in Experiment 1-3 indicated that the spatial instability of rivalry, measured as the fraction mixed percepts, is most likely enhanced after adaptation to a combination of monocular contrast transients and perceptual orientation transients, but not by monocular orientation transients and not by the presence of orientation conflict between the eyes. To further support this interpretation and to determine the degree of contribution of each individual transient type, we created a step-wise general linear model with the three transient types: monocular contrast (MC), perceptual orientation (PO), and monocular orientation (MO) as well as conflict (C) as predictors of the fraction mixed percepts (for input values; see Supplementary Table 4). The model also included an experiment-dependent intercept $\alpha$ to take into account variance created by differences in the groups of participants across experiments. The fraction mixed percept of the conflict condition, which was included in each experiment, served as the intercept $\alpha$ (Experiment 1: $M=0.36$; Experiment 2: $M=0.43$; Experiment 1: $\mathrm{M}=0.37$ ). The fitted model predicted the results very well, with a root mean squared error (RMSE) of $3 \%$ and an $r^{2}$ of 0.95 (Fig. 5). The betas (i.e., slopes) of the factors monocular orientation transients $(\beta=0.004, p=0.824)$ 
Two-factor model; RMSE: $3 \%$; $\mathrm{r}^{2}=\mathbf{0 . 9 5}$

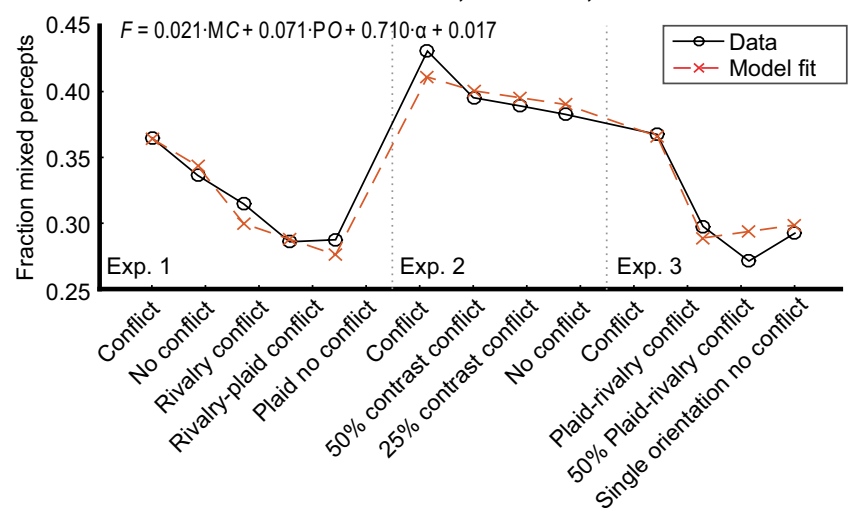

Figure 5. General linear model results. Modelled fraction mixed percepts (dashed red crosses) across the conditions for all experiments as compared to ground truth results (solid black circles) with the factors monocular contrast and perceptual orientation (and an intercept per experiment). The formula is the result of a general linear model with $F$ as fraction mixed percepts, $C$ as the presence (1) or absence ( 0 ) of monocular contrast, $O$ as the presence or absence of perceptual orientation transients, and $\alpha$ as the fraction mixed percepts of the conflict condition per experiment (see most left panels in plots (d) in Figs. 2-4) that served as an intercept to take into account group differences across experiments.

and conflict $(\beta=0.005, p=0.657)$ were not significant and therefore removed from the model. The final model's betas for monocular contrast $\left(\beta_{\mathrm{mc}}=0.021, p=0.041\right)$, perceptual orientation $\left(\beta_{\mathrm{po}}=0.071, p<0.001\right)$ transients, and experiment-dependent intercept $\left(\beta_{\mathrm{a}}=0.710, p=0.001\right)$ were significant. We conclude from this model that the presence of transients during the adaptation phase, whether produced by a change in grating orientation or contrast, and whether perceived or not, adapted and weakened interocular suppression, and disrupted the spatial coherence of the percept in subsequent binocular rivalry.

\section{General discussion}

With a set of three experiments we have assessed whether the precedence of mixed percepts during BR is affected by adaptation to the frequency and strength of stimulus transients or to the degree of interocular conflict as suggested by previous research. The visual transients during adaptation consisted of changes in monocular contrast, perceptual (binocular) orientation, and monocular orientation as a function of time. The fraction mixed percepts, used as a proxy of the degree of the weakening of interocular suppression and spatial destabilization of BR, showed a pattern across a total of 9 distinct conditions that was almost perfectly explained by incidence rates of monocular contrast and perceptual orientation transients. Monocular orientation transients and the presence of a conflict between the eyes as defined in previous work ${ }^{34}$ did not explain variance in the pattern of fraction mixed percepts to that degree. We conclude that visual transients affect the depth of interocular suppression during adaptation, resulting in weak, shallow, spatially incoherent binocular rivalry thereafter. Even though monocular contrast transients were inherent to conflict between the eyes in one critical condition (i.e., a blank in one eye and an oriented grating in the other eye), the fact that perceptual orientation transients affected the fraction mixed percepts in the absence of conflict, deems the explanation of visual transients the most parsimonious.

The question remains how transients relate to interocular suppression. We suggest that exogenous, involuntary attention may mediate the link between transients and the adaptation of interocular suppression. Visual transients, caused by changes in features content (e.g., orientation shifts) or eye-movements, alter perception and attention $^{26,27,38-41,45-49}$. Even subtle transients (i.e., cues) to one eye automatically draw attention and can bias perceptual dominance towards that eye $\mathrm{e}^{3,50-55}$. Similarly, subtle difference between the eyes also attracts attention, as demonstrated with a change blindness ${ }^{56}$ and visual search paradigm ${ }^{57-59}$. As dominance of both eyes is strengthened when attention is drawn to both eyes, the mutual, reciprocal suppression between the eyes is also strengthened $^{60}$. Alternatively, as attention is drawn to the flickering stimuli, their representations adapt and weaken ${ }^{61}$, exerting only weak suppression during rivalry thereafter. Our suggestion, therefore, is the following: the (visual) transients during the adaptation phase attract attention towards the images and, as a result, increase their mutual inhibition (and thus the amount of interocular suppression) and/or adapt the features present in the images. As a result, the strength of mutual inhibition between these images is decreased after adaptation, leading to more shallow rivalry (and hence more mixed percepts) during the following adaptation phase. An alternative explanation is related to working memory. Sterzer $\&$ Rees $^{62}$ identified a brain network including parietal and prefrontal areas involved in working memory to become active when dominance in binocular rivalry was temporally stabilized using intermittent blank presentations as strong transients. In line with this knowledge and an initial proposal ${ }^{36}$, they suggested that the sudden disappearance of an image during binocular rivalry activates mnemonic processes dedicated to hold the previously seen image in memory and prioritize it for visual awareness the moment it reappears. This memory process is not restrained to only the most recent image but likely holds and biases perception based on images that are observed for at least the last sixty seconds ${ }^{63}$. As an image is prioritized, it will also exert stronger suppression to the rivalling image. As the case in the current study, when both images are subject to 
transients, both will be prioritized and will mutually inhibit each other, that is, strengthen interocular suppression and proliferate its adaptation.

It is not unlikely that the effects of working memory and attention on interocular suppression interact. The sudden aspect of transients may (involuntarily and unconsciously) both draw attention and strengthen the (mnemonic) representations of previously seen images, therewith enhancing their inhibitory influence on competing images. However, neither explanation requires adaptation of a specialized conflict detection mechanism. In the model put forth in Said and Heeger, this mechanism is based on the idea of ocular opponency neurons ${ }^{35,64,65}$. Although such neurons appear likely candidates for involvement in binocular rivalry, and the initial prediction of the model by Said and Heeger that included a conflict detection mechanism explained their data well, the results reported here cannot be unified under that model. As such, we currently see no evidence that mechanisms based on ocular opponency neurons should be included in models of binocular rivalry.

It is important to note that in our study the intermittent presentation of blanks had a stronger effect on adaptation than the intermittent presentation of plaids. A similar effect has been reported before ${ }^{66}$, showing that the presentation of interleaved blanks enhanced the temporal stabilization of rivalry more than plaids. As blanks are more distinct from the orthogonal images and therefore more conspicuous, it makes sense that intermittent presentation of blanks adapted interocular suppression stronger than plaids. This conclusion may appear at odds with our observation that the monocular contrast transients (i.e., blanks) disrupted the spatial coherence of rivalry slightly weaker than perceptual orientation transients. Note however that the monocular contrast transients were not visible but the perceptual orientation transients were visible to the participant. As the visibility of transients is positively linked to the degree of drawing attention exogenously ${ }^{67}$ and the suppressive strength of an evoked traveling dominance wave ${ }^{27}$, it is not unexpected that the perceptually visible orientation transients adapted interocular suppression most. Our observation that a relatively high rate of orientation transients (e.g., see rivalry condition) increased the fraction mixed percepts more than a relatively low rate (e.g., see plaid-rivalry condition) further confirms the modulatory effect of transient visibility on the adaptation of interocular suppression. Although out of the scope of the current study, it would be interesting to investigate whether perceptual (and thus visible) contrast transients adapt interocular suppression to a similar degree as the perceptual orientation transients that we investigated here. A useful paradigm to test this would be intermittent presentation in which conflicting gratings disappear and appear as a function of time ${ }^{36,68}$.

To conclude, perceptual stability as expressed in the precedence of mixed percepts and traveling waves during rivalry is weakened when the eyes are stimulated beforehand with many, strong transients. Future work may shed light on the effect of visible and invisible transients on maintaining and adapting to visual representations.

Received: 17 December 2019; Accepted: 7 May 2020;

Published online: 26 May 2020

\section{References}

1. Blake, R. \& Logothetis, N. K. Visual competition. Nat Rev Neurosci 3, 13-21, https://doi.org/10.1038/nrn701 (2002).

2. Brascamp, J. W., Klink, P. C. \& Levelt, W. J. M. The 'laws' of binocular rivalry: 50 years of Levelt's propositions. Vision research 109, 20-37 (2015).

3. Mitchell, J. F., Stoner, G. R. \& Reynolds, J. H. Object-based attention determines dominance in binocular rivalry. Nature 429, 410 (2004).

4. Pasley, B. N., Mayes, L. C. \& Schultz, R. T. Subcortical discrimination of unperceived objects during binocular rivalry. Neuron 42, 163-172 (2004).

5. Brascamp, J. W. \& Blake, R. Inattention abolishes binocular rivalry: Perceptual evidence. Psychological Science 23, 1159-1167 (2012).

6. Paffen, C. L., Alais, D. \& Verstraten, F. A. Attention speeds binocular rivalry. Psychol Sci 17, 752-756, https://doi.org/10.1111/j.14679280.2006.01777.x (2006)

7. Paffen, C. L., Plukaard, S. \& Kanai, R. Symbolic magnitude modulates perceptual strength in binocular rivalry. Cognition 119, 468-475 (2011)

8. Frässle, S., Sommer, J., Jansen, A., Naber, M. \& Einhäuser, W. Binocular rivalry - frontal activity relates to introspection and action, but not to perception. Journal of Neuroscience 34, 1738-1747 (2014).

9. Leopold, D. A. \& Logothetis, N. K. Multistable phenomena: changing views in perception. Trends Cogn Sci 3, 254-264 (1999).

10. Tong, F., Meng, M. \& Blake, R. Neural bases of binocular rivalry. Trends Cogn Sci 10, 502-511, https://doi.org/10.1016/j. tics.2006.09.003 (2006).

11. Engel, A. K., Fries, P., König, P., Brecht, M. \& Singer, W. Temporal binding, binocular rivalry, and consciousness. Consciousness and cognition 8, 128-151 (1999).

12. Jiang, Y., Costello, P., Fang, F., Huang, M. \& He, S. A gender-and sexual orientation-dependent spatial attentional effect of invisible images. Proceedings of the National Academy of Sciences 103, 17048-17052 (2006).

13. Lin, Z. \& He, S. Seeing the invisible: The scope and limits of unconscious processing in binocular rivalry. Nature Precedings, 1-1 (2008).

14. Carmel, D., Arcaro, M., Kastner, S. \& Hasson, U. How to create and use binocular rivalry. JoVE (Journal of Visualized Experiments), e2030 (2010)

15. Julesz, B. \& Miller, J. E. Independent spatial-frequency-tuned channels in binocular fusion and rivalry. Perception 4, 125-143 (1975).

16. Blake, R. \& Boothroyd, K. The precedence of binocular fusion over binocular rivalry. Perception \& Psychophysics 37, 114-124 (1985).

17. Wolfe, J. M. Influence of spatial frequency, luminance, and duration on binocular rivalry and abnormal fusion of briefly presented dichoptic stimuli. Perception 12, 447-456 (1983).

18. Riesen, G., Norcia, A. M. \& Gardner, J. L. Humans perceive binocular rivalry and fusion in a tristable dynamic state. Journal of Neuroscience 39, 8527-8537 (2019).

19. Moreau, G. In 2013 26th Conference on Graphics, Patterns and Images Tutorials. 6-14 (IEEE).

20. Nelson, J. I. Globality and stereoscopic fusion in binocular vision. Journal of Theoretical Biology 49, 1-88 (1975).

21. Naber, M., Frässle, S. \& Einhäuser, W. Perceptual rivalry: reflexes reveal the gradual nature of visual awareness. PLoS One 6, e20910, https://doi.org/10.1371/journal.pone.0020910 (2011).

22. Lee, S. H., Blake, R. \& Heeger, D. J. Traveling waves of activity in primary visual cortex during binocular rivalry. Nat Neurosci 8 , 22-23, https://doi.org/10.1038/nn1365 (2005).

23. Kang, M. S. \& Blake, R. An integrated framework of spatiotemporal dynamics of binocular rivalry. Front Hum Neurosci 5, 88, https:// doi.org/10.3389/fnhum.2011.00088 (2011). 
24. Wilson, H. R., Blake, R. \& Lee, S. H. Dynamics of travelling waves in visual perception. Nature 412, 907-910, https://doi. org/10.1038/35091066 (2001).

25. Knapen, T., van Ee, R. \& Blake, R. Stimulus motion propels traveling waves in binocular rivalry. PLoS One 2, e739, https://doi. org/10.1371/journal.pone.0000739 (2007).

26. Paffen, C. L., Naber, M. \& Verstraten, F. A. The spatial origin of a perceptual transition in binocular rivalry. PLoS One 3, e2311, https://doi.org/10.1371/journal.pone.0002311 (2008).

27. Naber, M., Carter, O. \& Verstraten, F. A. Suppression wave dynamics: visual field anisotropies and inducer strength. Vision Res 49, 1805-1813, https://doi.org/10.1016/j.visres.2009.04.017 (2009).

28. Stuit, S. M., Verstraten, F. A. \& Paffen, C. L. Saliency in a suppressed image affects the spatial origin of perceptual alternations during binocular rivalry. Vision Res 50, 1913-1921, https://doi.org/10.1016/j.visres.2010.06.014 (2010).

29. Arnold, D. H., James, B. \& Roseboom, W. Binocular rivalry: Spreading dominance through complex images. Journal of Vision 9, 4-4 (2009).

30. Kang, M. S., Lee, S. H., Kim, J., Heeger, D. \& Blake, R. Modulation of spatiotemporal dynamics of binocular rivalry by collinear facilitation and pattern-dependent adaptation. Journal of vision 10, 3-3 (2010).

31. Blake, R., O'Shea, R. P. \& Mueller, T. J. Spatial zones of binocular rivalry in central and peripheral vision. Vis Neurosci 8, 469-478 (1992).

32. Knapen, T., Kanai, R., Brascamp, J., van Boxtel, J. \& van Ee, R. Distance in feature space determines exclusivity in visual rivalry. Vision Res 47, 3269-3275, https://doi.org/10.1016/j.visres.2007.09.005 (2007).

33. Alais, D. \& Melcher, D. Strength and coherence of binocular rivalry depends on shared stimulus complexity. Vision research 47, 269-279 (2007)

34. Said, C. P. \& Heeger, D. J. A model of binocular rivalry and cross-orientation suppression. PLoS computational biology 9, e1002991 (2013).

35. Poggio, G. F. \& Talbot, W. H. Mechanisms of static and dynamic stereopsis in foveal cortex of the rhesus monkey. The Journal of physiology 315, 469-492 (1981).

36. Leopold, D. A., Wilke, M., Maier, A. \& Logothetis, N. K. Stable perception of visually ambiguous patterns. Nat Neurosci 5, 605-609, https://doi.org/10.1038/nn851 (2002).

37. Orbach, J., Ehrlich, D. \& Heath, H. A. Reversibility of the Necker cube: I. An examination of the concept of "satiation of orientation". Perceptual and motor skills 17, 439-458 (1963).

38. Kanai, R. \& Kamitani, Y. Time-locked perceptual fading induced by visual transients. J Cogn Neurosci 15, 664-672 (2003).

39. Kanai, R., Moradi, F., Shimojo, S. \& Verstraten, F. A. Perceptual alternation induced by visual transients. Perception 34, 803-822 (2005).

40. Murata, T., Matsui, N., Miyauchi, S., Kakita, Y. \& Yanagida, T. Discrete stochastic process underlying perceptual rivalry. Neuroreport 14, 1347-1352 (2003).

41. Levelt, W. J. The alternation process in binocular rivalry. British Journal of Psychology 57, 225-238 (1966).

42. Wheatstone, C. Contributions to the physiology of vision. Part the first: On some remarkable, and hitherto unobserved, phænomena of binocular vision. Philos. Trans. R. Soc. Lond. 128, 371-394 (1838).

43. Brascamp, J. W. \& Naber, M. Eye tracking under dichoptic viewing conditions: a practical solution. Behavior research methods 49, 1303-1309 (2017).

44. Webster, M. A. Visual adaptation. Annual review of vision science 1, 547-567 (2015).

45. Posner, M. I. \& Cohen, Y. Components of visual orienting. Attention and performance X: Control of language processes 32, 531-556 (1984).

46. Theeuwes, J., Kramer, A. F., Hahn, S. \& Irwin, D. E. Our eyes do not always go where we want them to go: Capture of the eyes by new objects. Psychological Science 9, 379-385 (1998).

47. Atchley, P., Kramer, A. F. \& Hillstrom, A. P. Contingent capture for onsets and offsets: Attentional set for perceptual transients. Journal of Experimental Psychology: Human Perception and Performance 26, 594 (2000).

48. Steinman, B. A., Steinman, S. B. \& Lehmkuhle, S. Research note transient visual attention is dominated by the magnocellular stream. Vision research 37, 17-23 (1997).

49. Wilke, M., Logothetis, N. K. \& Leopold, D. A. Generalized flash suppression of salient visual targets. Neuron 39, 1043-1052 (2003).

50. Hancock, S. \& Andrews, T. J. The role of voluntary and involuntary attention in selecting perceptual dominance during binocular rivalry. Perception 36, 288-298 (2007).

51. Paffen, C. L. \& Van der Stigchel, S. Shifting spatial attention makes you flip: Exogenous visual attention triggers perceptual alternations during binocular rivalry. Atten Percept Psychophys 72, 1237-1243, https://doi.org/10.3758/APP.72.5.1237 (2010).

52. Ooi, T. L. \& He, Z. J. Binocular rivalry and visual awareness: The role of attention. Perception 28, 551-574 (1999).

53. Paffen, C. L. \& Alais, D. Attentional modulation of binocular rivalry. Frontiers in Human Neuroscience 5, 105 (2011).

54. Dieter, K. C., Melnick, M. D. \& Tadin, D. When can attention influence binocular rivalry? Attention, Perception, \& Psychophysics 77, 1908-1918 (2015).

55. de Graaf, T. A., van Ee, R., Croonenberg, D., Klink, P. C. \& Sack, A. T. Visual suppression at the offset of binocular rivalry. Journal of Vision 17, 2-2 (2017).

56. Paffen, C. L., Hessels, R. S. \& Van der Stigchel, S. Interocular conflict attracts attention. Attention, Perception, \& Psychophysics 74, 251-256 (2012)

57. Wolfe, J. M. \& Franzel, S. L. Binocularity and visual search. Perception \& Psychophysics 44, 81-93 (1988).

58. Paffen, C. L., Hooge, I. T. C., Benjamins, J. S. \& Hogendoorn, H. A search asymmetry for interocular conflict. Attention, Perception, \& Psychophysics 73, 1042-1053 (2011).

59. Zou, B., Utochkin, I. S., Liu, Y. \& Wolfe, J. M. Binocularity and visual search-Revisited. Attention, Perception, \& Psychophysics 79, 473-483 (2017).

60. Tsuchiya, N., Koch, C., Gilroy, L. A. \& Blake, R. Depth of interocular suppression associated with continuous flash suppression, flash suppression, and binocular rivalry. Journal of vision 6, 6-6 (2006).

61. Anstis, S. Contour adaptation. Journal of Vision 13, 25-25 (2013)

62. Sterzer, P. \& Rees, G. A neural basis for percept stabilization in binocular rivalry. Journal of cognitive neuroscience 20, 389-399 (2008).

63. Brascamp, J. W. et al. Multi-timescale perceptual history resolves visual ambiguity. PLoS One 3, e1497, https://doi.org/10.1371/ journal.pone.0001497 (2008).

64. Ohzawa, I. \& Freeman, R. D. The binocular organization of simple cells in the cat's visual cortex. Journal of Neurophysiology 56, 221-242 (1986)

65. Smith, E. L., Chino, Y. M., Ni, J., Ridder, W. H. \& Crawford, M. L. J. Binocular spatial phase tuning characteristics of neurons in the macaque striate cortex. Journal of Neurophysiology 78, 351-365 (1997).

66. Kanai, R., Knapen, T. H., van Ee, R. \& Verstraten, F. A. Disruption of implicit perceptual memory by intervening neutral stimuli. Vision Res 47, 2675-2683, https://doi.org/10.1016/j.visres.2007.06.016 (2007).

67. Fuller, S., Park, Y. \& Carrasco, M. Cue contrast modulates the effects of exogenous attention on appearance. Vision Research 49, 1825-1837 (2009).

68. Maier, A., Wilke, M., Logothetis, N. K. \& Leopold, D. A. Perception of temporally interleaved ambiguous patterns. Curr Biol 13, 1076-1085 (2003). 


\section{Author contributions}

M.N., S.S., Y.D.K., and C.P. designed the experiments. Author Y.D.K. collected the data. M.N., S.S., and Y.D.K. programmed the experiments and analyzed data. Author M.N. \& C.P. wrote the concept paper and S.S., Y.D.K., and S.V.d.S. contributed to the final paper.

\section{Competing interests}

The authors declare no competing interests.

\section{Additional information}

Supplementary information is available for this paper at https://doi.org/10.1038/s41598-020-65678-7.

Correspondence and requests for materials should be addressed to M.N.

Reprints and permissions information is available at www.nature.com/reprints.

Publisher's note Springer Nature remains neutral with regard to jurisdictional claims in published maps and institutional affiliations.

(c) (i) Open Access This article is licensed under a Creative Commons Attribution 4.0 International License, which permits use, sharing, adaptation, distribution and reproduction in any medium or format, as long as you give appropriate credit to the original author(s) and the source, provide a link to the Creative Commons license, and indicate if changes were made. The images or other third party material in this article are included in the article's Creative Commons license, unless indicated otherwise in a credit line to the material. If material is not included in the article's Creative Commons license and your intended use is not permitted by statutory regulation or exceeds the permitted use, you will need to obtain permission directly from the copyright holder. To view a copy of this license, visit http://creativecommons.org/licenses/by/4.0/.

(C) The Author(s) 2020 